\title{
Categorías de un modelo andragógico para los cuidadores de enfermos de Alzheimer
}

\author{
Towards improving an Andragogic learning Model for professional \\ taking care of Alzheimer Patients.
}

\section{Resumen}

Introducción. La andragogía está orientada hacia la educación de los adultos; los cuidadores como adulto responsables de un enfermo de Alzheimer deben contar con herramientas didácticas que mejoren su calidad de vida y desempeño.

Objetivo. Determinar el enfoque para un modelo andragógico que incremente la capacidad del cuidador de un enfermo de Alzheimer basado en el significado de su experiencia.

Metodología. Se trata un estudio observacional, descriptivo, exploratorio; la muestra fue de 29 cuidadores de enfermos de Alzheimer, siendo adultos, mujeres y familiares cercanos del paciente. A dichos cuidadores se le aplicó un cuestionario de 22 ítems de sobrecarga del cuidador propuesta por Zarit sobre la relación del cuidador con el enfermo, las relaciones sociales del cuidador, el efecto del cuidado en el estrés, capacidad y su salud, teniendo un alfa de Cronbach de .93.
Resultados. Los resultados del estudio señalaron que el modelo andragógico debe orientarse de manera particular hacia conocimientos, habilidades e información que forme e instruya al cuidador sobre su capacidad para hacer frente al cuidado de un enfermo de Alzheimer, elementos como salud, relaciones con el paciente y con otras personas son afectados por razón del cuidado.

De los resultados de otras investigaciones donde está presente un cuidador y un paciente, el presente documento coincide en señalar una incidencia sobre la vida cotidiana del cuidador por efecto de las relaciones y la gravedad de la enfermedad del paciente.

Conclusiones: Es factible diseñar un modelo andragógico fundamentado en: conocimientos, habilidades e información que instruyan al cuidador sobre su capacidad para hacer frente al cuidado de un enfermo de Alzheimer.

Palabras clave: andragogía, sentido de vida, cuidador, Alzheimer, relaciones, capacidad. 


\section{Abstract}

Andragogic learning models are oriented towards adults taking care of Alzheimer patients. This study has the objective of focusing on the improvement of an andragogic learning model so that it promotes and enhances the experience and capacity of professional taking care of Alzheimer patients.

Methodology: Basic, observational, descriptive, and exploratory study. The sample was formed with 29 adults taking care of Alzheimer patients. These adults completed a 22 item questionnaire related to: work overload issves as viewed by zarit; social relations; stress impacts; and capacity. Cronbach alpha turned out to be .93

Results: Showed that this andragogic Learning model should be oriented towards knowledge, skills and information which prepare the professional to face the challenge of taking care of Alzheimer patients. Finding of this study were similar to those reported in other studies.

\section{Conclusions:}

It is possible to deseng on andragogic learning model gased on: knowledge, skills, and information which prepare the professional to face the challenge of taking care Alzheimer patients.

Key words: Andragogic learning model, sense of life, health care, alzheimer

\section{INTRODUCCIÓN}

La andragogía, como arte y ciencia de la enseñanza, se ha formalizado de manera reciente a favor de la educación de los adultos. Estando inmersa en la educación permanente e incluso en la educación no formal o no estructurada, la andragogía se desarrolla a través de principios de participación y horizontalidad, donde los alumnos y los maestros-facilitadores tienen relación horizontal no jerárquica.

En particular, la andragogía ofrece principios fundamentales del aprendizaje de adultos, donde se permite diseñar y guiar procesos docentes más eficaces y orientados hacia un participante determinado. Se trata de un modelo transaccional, ya que remite a las características de aprendizaje, no a las metas ni a los objetivos. Igualmente, se puede aplicar a cualquier contexto de enseñanza para adultos determinados, tal como los cuidadores familiares de enfermos de Alzheimer.

La andragogía está orientada hacia la educación de los adultos. Considerando la diversidad de antecedentes, grupos, formación, problemáticas y objetivos, la andrago-

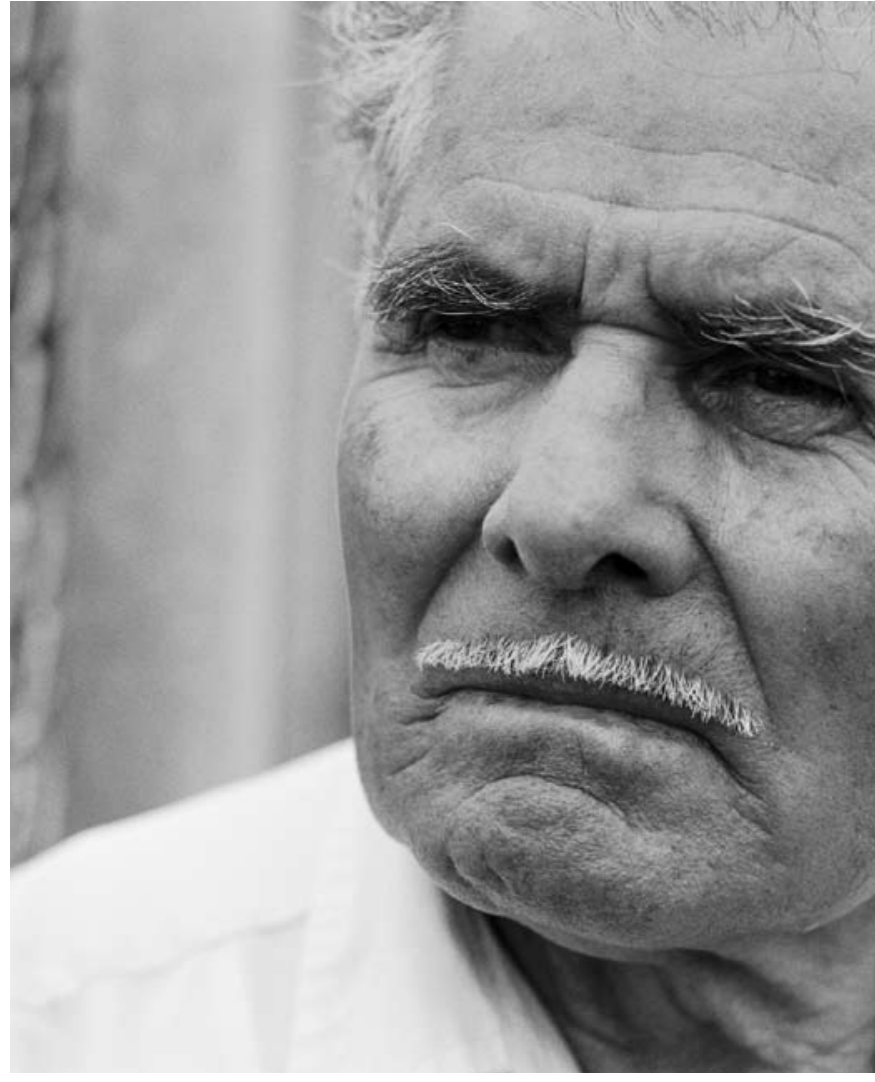


gía toma en cuenta la necesidad y motivación particular de aprender, de aplicar, de solucionar o de emprender cambios basados en el conocimiento.

Un grupo definido, tal como los adultos cuidadores familiares de enfermos de Alzheimer, enfrentan una situación que incide en sus relaciones con el enfermo, con sus demás familiares y amigos, consigo mismos o con la cualquier otra persona.

Ante la identificación del sujeto, de su circunstancia, de sus afectaciones físicas, psíquicas y emocionales, es necesario basada en el sentido de vida, determinar cuáles son sus necesidades educativas para minimizar los riesgos que impacten en su salud, en sus relaciones y en su desempeño general.

De ello, el propósito de esta investigación es identificar el enfoque de debe contener un modelo andragógico para cuidadores de enfermos de Alzheimer. Este modelo se justifica en la medida de disponer de una herramienta didáctica que permita al cuidador incrementar su calidad de vida y reorientar su desempeño.

\section{Las premisas andragógicas:}

- La necesidad de saber: Todo adulto necesita saber porqué debe aprender algo antes de aprenderlo.

- El autoconcepto de los alumnos. Los adultos tienen un autoconcepto de seres responsables de sus propias acciones, de su propia vida, tienen necesidad de ser considerados y tratados como capaces de dirigirse, resienten y se resisten a las situaciones en que otros les imponen su voluntad.

- El papel de la experiencia de los alumnos. El acento en educación de adultos está en la individualización de la enseñanza y las estrategias de aprendizaje.

- Disposición para aprender. Los adultos están dispuestos a aprender lo que necesitan saber y sean capaces de hacer con el propósito de enfrentar las situaciones de la vida real.

- Orientación del aprendizaje. Los adultos se centran en la vida, se motivan a aprender en la medida que perciban que el aprendizaje les ayudará en su desempeño y a tratar con los problemas de la vida, obtienen conocimientos, destrezas, valores y actitudes, de una manera más certera en cuanto se le presentan algunas situaciones de la vida real.

- Motivación. Los adultos responden a algunos motivadores externos. ${ }^{1}$
La necesidad de conocer al alumno, como principio central que establece que los adultos necesitan conocer las razones para comprometerse a un aprendizaje, ha llevado a la premisa de que los adultos deben participar en un proceso de planeación de su aprendizaje.

Al hacer participar a los adultos como colaboradores se satisface su necesidad de conocer y se estimula su autoconcepto como alumnos independientes, dicha premisa implica: necesidad de saber cómo transcurrirá el aprendizaje, que aprendizaje ocurrirá y por qué este es importante.

\section{¿Quién es el cuidador?}

En relación al cuidador, su actividad no está exenta de repercusiones para su salud y bienestar. Diversos autores especialmente desde el ámbito de las ciencias sociales y médicas han estudiado esta problemática y han puesto de manifiesto la sobrecarga física y emocional que presenta los cuidadores familiares pudiendo manifestarse en que el cuidador se convierta en un enfermo secundario. ${ }^{2}$

El cuidado en la casa a un familiar tiene altos costos. Se ha establecido que el cuidador está expuesto a las dificultades económicas y que su trabajo lo hace vulnerable al aislamiento y a la exclusión social. Los síntomas depresivos pueden ser hasta del $40 \%$ en el total de los cuidadores, quienes se perciben con mala salud y una sensación de cansancio físico grande. El costo económico es difícil de calcular; incluso se afirma que, en algunas enfermedades ningún Estado puede asumir dicho costo.

Dentro de la sintomatología de los cuidadores es posible encontrar complicaciones físicas, mentales y sociofamiliares, ${ }^{3}$

- Complicaciones físicas: Dolores crónicos, cefaleas tensionales, fatiga crónica, trastornos digestivos (dispepsias), pérdida o incremento de peso corporal, infecciones virales a repetición (ejemplo: gripes) por inmunodepresión, úlcera gastrointestinal, quejas somáticas: dolor crónico del aparato locomotor, astenia, alteración del ciclo sueño-vigilia, deterioro de la función inmune, mayor predisposición a úlcera péptica, patología cardiovascular, entre otras.

Complicaciones mentales o emocionales: ansiedad/ estrés (en el 50\% de los casos), depresión (es el pro- 
blema más frecuente), insomnio, falta de energía, sentimientos de pérdida de control, sentimientos de culpa, aparición de excesivo uso del cinismo y o sarcasmo, sobreinvolucración con el paciente, insomnio, alta tasa de automedicación (psicofármacos y analgésicos). ${ }^{4}$

- Conflictos sociofamiliares: problemas familiares, conflictos laborales, altercados con sus mismos compañeros, aislamiento social, disminución de las actividades de ocio y recreación, dificultades económicas debidas a los bajos salarios o a la falta de una remuneración.

- En general, los cuidadores no combaten la enfermedad crónica sino que la manejan, lo cual es una actividad que implica más de lo estrictamente médico, y por lo general, permanece invisible para el personal de salud.

El cuidado en la casa requiere que los cuidadores tengan destrezas y conocimientos cada vez más complejos, y a medida que la enfermedad avanza, las relaciones familiares van cambiando, aumentan las complicaciones de la enfermedad y el sufrimiento de quienes cuidan.

Ante este contexto y en el marco de la andragogía es necesario determinar la problemática desde la perspectiva del mismo cuidador, explorando e indagando sobre qué le afecta, cómo le afecta y qué debe hacerse para contrarrestarlo.

En este sentido, en busca de elementos que definan un modelo andragógico que considere en primer lugar la necesidad del adulto cuidador e incorpore en su enseñanza aquello que le será útil para mejorar su calidad y sentido de vida, ${ }^{1}$ se ha aplicado un cuestionario a un grupo determinado de cuidadores adultos.

\section{METODOLOGÍA}

Se trata un estudio observacional, descriptivo, exploratorio, realizado en el periodo 2009-1 y 2009-2 en el cual se aplico un cuestionario cerrado a 29 cuidadores familiares de enfermos de Alzheimer, la elección de los cuidadores fue convencional. Se utilizó el cuestionario de Zarit de 22 preguntas sobre la relación del cuidador con el enfermo, las relaciones sociales del cuidador, el efecto del cuidado en el estrés, capacidad y su salud. Con dicha escala se determina el grado de impacto del cuidado sobre el cuidador de un enfermo de Alzheimer, los 29 cuidadores aceptaron participar de manera voluntaria. Los resultados de este sondeo dan una idea de las áreas donde es posible dirigir la atención para estructurar un modelo andragógico de educación para el cuidador.

La escala de las preguntas tuvo 5 niveles: nunca, casi nunca, a veces, frecuentemente y casi siempre. Para cada nivel se determinó la frecuencia absoluta y relativa. El Alpha de Crombach es de 0.93. En la codificación se agruparon preguntas que correspondieron a 5 grupos: relación con el paciente, relaciones sociales, estrés, capacidad y salud.

\section{RESULTADOS}

Los resultados obtenidos son los siguientes: Gráfica $n^{\circ} 1$. Distribución de respuestas obtenidas por los cuidadores en base a si la persona cuidada solicita más ayuda de la que realmente necesita. 2008

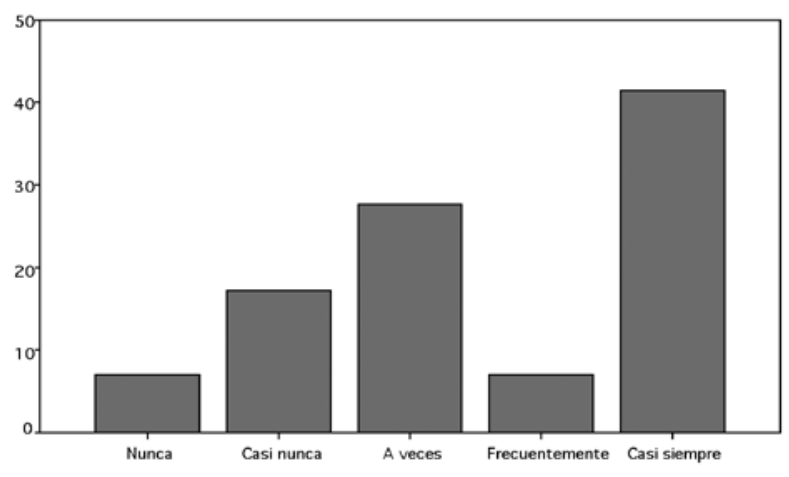

Más ayuda de la necesaria

\section{Grafica $n^{\circ} 2$}

A causa del tiempo que gasta con su familiar no tiene tiempo suficiente para el/ella misma/o.

2008

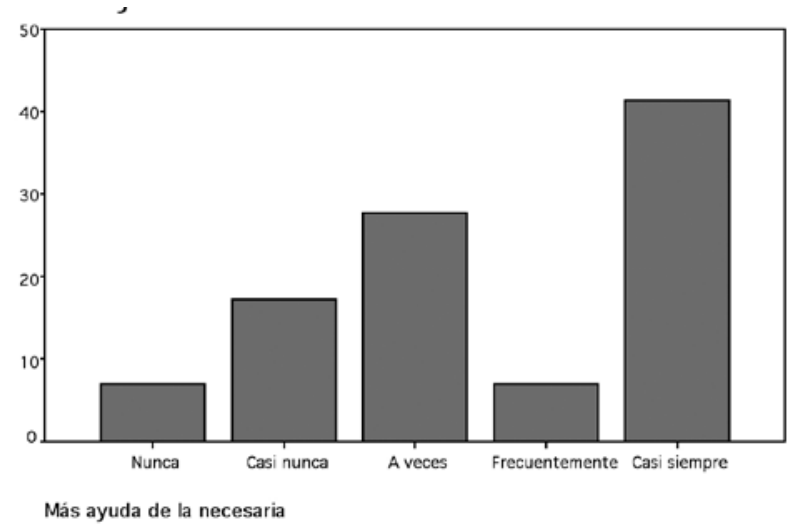


En relación a las respuestas del cuidador sobre el paciente, los resultados indican que el enfermo de Alzheimer demanda más cuidado del que necesita, correspondiéndose con el reconocimiento del cuidador de que su familiar depende de él.

En este grupo, con un total de 87 respuestas posibles (29 cuidadores por 3 preguntas), en los rangos de frecuentemente y casi siempre ocurre un total de 49 respuestas, representada por el $56 \%$ de los cuidadores.

En el extremo, los cuidadores que señalan que nunca y casi nunca el enfermo necesita más ayuda de la que requiere y no depende del cuidador, representa el $21 \%$.

\section{Grafica $n^{\circ} 2$. A causa del tiempo que gasta con su familiar no tiene tiempo suficiente para el/ella misma/o. 2008}

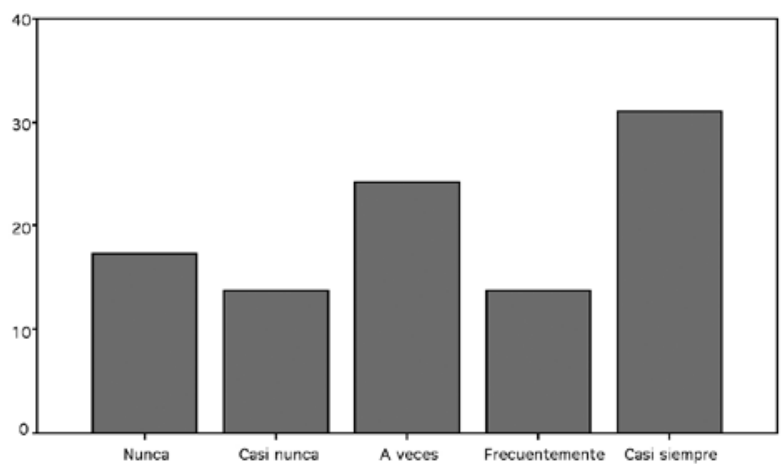

Tiempo personal suficiente

De las respuestas sobre las relaciones sociales del cuidador, señalan que el cuidador no ve afectada sus relaciones sociales a causa del cuidado del enfermo.

En este grupo, con un total de 145 respuestas posibles (29 cuidadores por 5 preguntas), en los rangos de nunca y casi nunca ocurre un total de 75 respuestas, representada por el $52 \%$ de los cuidadores.

En el extremo, los cuidadores que señalan que frecuentemente y casi siempre a causa del cuidado del enfermo se ven afectadas sus relaciones, representa el $29 \%$.

\section{Gráfica $n^{\circ}$ 3. Estresado por cuidar a su familia, además de atender otras responsabilidades. 2008}

En relación al estrés, representado por vergüenza, irritación, temor, agotamiento, pérdida de control y sobrecarga, la mayoría señala que los cuidadores en el $66 \%$ de ellos se estresan por causa del cuidado hacía su familiar.

En este grupo, con un total de 203 respuestas posibles (29 cuidadores por 7 preguntas), los cuidadores que señalan que nunca y casi nunca el cuidado del enfermo lo estresa, representa el 47\%.

En el extremo, en los rangos de frecuentemente y casi siempre ocurre un total de 55 respuestas, representada por el $27 \%$ de los cuidadores.

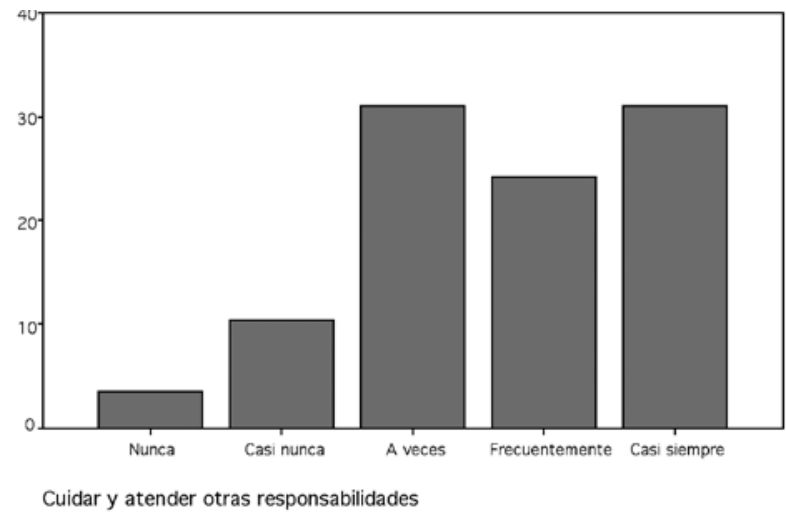

\section{Gráfica $n^{\circ} 4$}

Siente que no va a ser capaz de cuidar a su familiar durante mucho tiempo. 2008

En relación con la capacidad, representada por la disposición de dinero, inseguridad personal, capacidad para cuidar, delegación del cuidado y posibilidad de hacer más, existe una relativa homogeneidad en las respuestas, sin que exista definición sobre la frecuencia.

En este grupo, con un total de 174 respuestas posibles (29 cuidadores por 6 preguntas), el 52\% de los cuidadores menciona que frecuentemente y casi siempre sienten falta de capacidad para atender a su familiar enfermo,

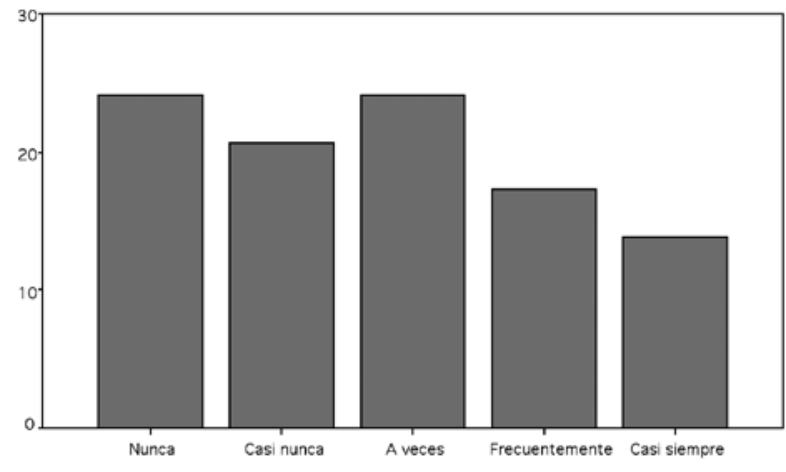

Siente que no puede cuidar más tiempo 
agregando un 38\% que señala que a veces se siente incapaz de poder cuidar a su enfermo.

\section{Gráfica $n^{\circ} 5$. Su salud se ha visto afectada por tener que cuidar a su familia. 2008}

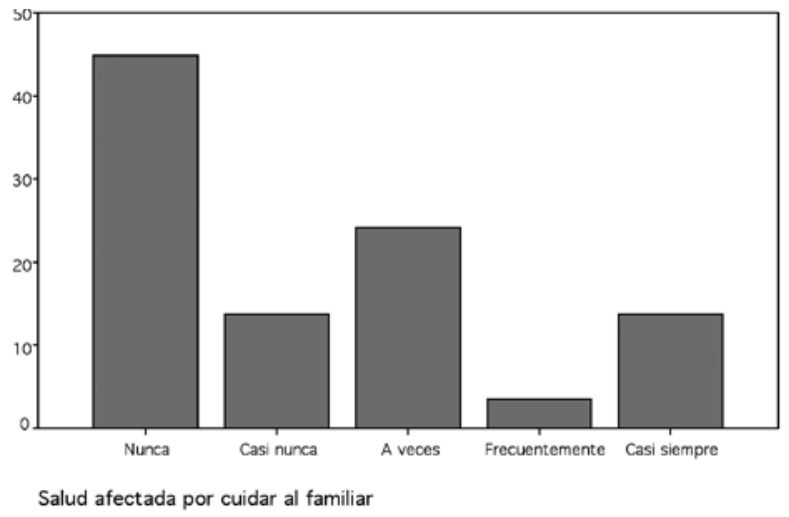

Respecto a la salud, de las 29 preguntas, el 45\% señala que su salud no se ve afectada por el cuidado, sin embargo, es importante señalar que el 56\% de las personas entrevistadas se encuentran en los rubros de casi nunca, a veces, frecuentemente y casi siempre, su salud se ve afectada.

\section{CONCLUSIONES}

En términos del objetivo general del cuestionario, relativo a determinar el grado de impacto del cuidado sobre el cuidador de un enfermo de Alzheimer, se concluye que:

- El cuidador familiar percibe que el paciente demanda más cuidado del que necesita, además de reconocer una situación de dependencia.

- No obstante lo demandante del cuidado que requiere un enfermo de Alzheimer, el cuidador no señala que se vean afectadas sus relaciones sociales.

- En correspondencia a lo anterior, el cuidador señala en mayor medida que el cuidado le genere estrés, tal como podría suponerse ante el contexto del cuidado de un enfermo de Alzheimer.

- Acerca de la capacidad del cuidador familiar, los resultados reflejan que existe una definición determinada en este grupo de estudio.

- En general, los cuidadores reportaron que su salud se ve afectada en mayor medida por el esfuerzo de cuidado.
De lo anterior, tomando en cuenta al grupo de estudio, el modelo andragógico debe orientarse de manera particular hacia conocimientos, habilidades e información que forme e instruya al cuidador sobre su capacidad para hacer frente al cuidado de un enfermo de Alzheimer, sin que se comprometa su relación con el paciente, sus relaciones sociales y su salud.

\section{REFERENCIAS BIBLIOGRÁFICAS}

1 Knowles, M. Andragogía. México: Oxford. 2001.

2 Cavadinha C, Labronici L, Herreira T. Sentirse impotente: un sentimiento expresado por cuidadores de víctimas de violencia sexual. Revista Latino-Americana de Enfermagem vol.17 No.3. Brasil. Mayo/Junio 2009

3 Hernández Z. Cuidadores del adulto mayor residente en asilos. México. Instituto de Investigaciones Psicológicas de la Universidad Veracruzana. 2006.

4 Molina L. El apoyo social como modulador de la carga del cuidador de enfermos de Alzheimer. En Psicología y Salud, Universidad Veracruzana. México, 2005, № Vol. enero-junio, año/vol. 15, número 001. 2005

\section{REFERENCIAS COMPLEMENTARIAS}

Balestrero Sandra, Demencias y Familia, Revista Argentina de Alzheimer. Argentina. 2000. p. 2

Borges G. El papel de la epidemiología en la investigación de los trastornos mentales. Salud Pública de México. México. 2004. p. 452

Creswell, J.W. Qualitative inquiry and research. Thousand Oaks. California Sage Publications. 1998

Crean taller-escuela para cuidadores de pacientes con Alzheimer. México: El Universal. 2008. 18 de septiembre URL disponible en: http://www.eluniversal. com.mx/articulos/49426.html

Estudio de red personal en cuidadores de pacientes con Alzheimer. México, Universidad Nacional Autónoma de México. 2007. URL disponible en: http:// revista-redes.rediris.es/webredes/vmesahispana/Teran.htm

\section{DIRECCIÓN PARA CORRESPONDENCIA}

Mtra. Ma. Antonieta Larios S.: aiueoeneo@hotmail.com 